\title{
High-Fat Diets-Induced Metabolic Alterations Alter the Differentiation Potential of Adipose Tissue-Derived Stem Cells ${ }^{*}$
}

\author{
Vikie Lamontagne ${ }^{1,2}$, Souhad El Akoum ${ }^{1,2}$, Isabelle Cloutier ${ }^{2}$, Jean-François Tanguay ${ }^{1,2 \#}$ \\ ${ }^{1}$ Département des Sciences Biomédicales, Université de Montréal, Montréal, Canada \\ ${ }^{2}$ Montreal Heart Institute, Montréal, Canada \\ Email: \#jean-francois.tanguay@icm-mhi.org
}

Received April 3, 2013; revised May 5, 2013; accepted June 5, 2013

Copyright (C) 2013 Vikie Lamontagne et al. This is an open access article distributed under the Creative Commons Attribution License, which permits unrestricted use, distribution, and reproduction in any medium, provided the original work is properly cited.

\begin{abstract}
Background: Adipose tissue-derived stem cells (ASC) possess the ability to differentiate into adipocytes or endothelial cells to help in the adipogenesis, vasculogenesis and vascular repair. This study aims at determining the impact of highfat diets (HFD)-induced type 2 diabetes (T2D) on the differentiation potential of ASC. Results: C57BL/6J male mice were fed a vegetal (VD) or an animal (AD) HFD. Isolation of ACS from mice showing different levels of metabolic alterations reveals that advanced T2D did not affect the number of cells per gram of tissue. Rather, a higher proportion of inflammatory CD36 ${ }^{+}$cells was identified in HFD fed mice. Despite a marked decreased expression of adipogenic genes (aP2, $\mathrm{C} / \mathrm{EBP} \alpha$ and PPAR $\gamma 2$ ), ASC from HFD groups had a higher adipogenic potential and a lower endothelial differentiation potential in vitro compared to control. ASC from the VD group had enhanced cyclin B1 expression and had more adipogenic potential compared to AD group. Conclusion: Our results demonstrate that the metabolic modifications, linked to the nature of fatty acids in diets, modulate the differentiation potential of ASC with increased adipogenesis to the detriment of the endothelial pathway. Results highlight the importance of evaluating the ASC differentiation behavior in a context of autologous cell-based therapy for the repair of vascular tissues in diabetic patients.
\end{abstract}

Keywords: Adipose Tissue-Derived Stem Cells; Type 2 Diabetes; Adipocytes; Endothelial Cells; Differentiation; Vascular Cellular Therapy

\section{Introduction}

Adipose tissue is an energy storage organ that has an endocrine role secreting a variety of bioactive hormones called adipokines [1]. It is also a niche for a large panel of multipotent Adipose tissue-derived Stem Cells (ASC) contributing to its roles as an energy storage and endocrine tissue. The commitments of ACS give rise to various cell types (myoblasts, osteoblasts, chondroblasts or adipoblasts) depending on the appropriate stimuli $[2,3]$. Among others, these cells will develop into preadipo-

\footnotetext{
${ }^{*}$ Vikie Lamontagne: conception and design, collection and/or assembly of data, data analysis and interpretation, manuscript writing. Souhad El Akoum: conception and design, PCR data, manuscript writing. Isabelle Cloutier: conception and design, data analysis and interpretation, manuscript writing. Jean-François Tanguay: conception and design, manuscript review, final approval of manuscript.

This project was supported financially by grants from the Fondation des maladies du Coeur du Québec and from the Fondation de l'Institut de Cardiologie de Montréal to Dr Jean-François Tanguay.

${ }^{\#}$ Corresponding author.
}

cytes and endothelial cells [4-7]. While preadipocytes giving birth to mature adipocytes helping the expansion and the development of adipose tissue, endothelial cells play an essential role in vascularization of expanded fat pad and the renewal of damaged vessels. Thus, adipose tissue constitutes an important niche of stem cells helping in the cell turnover and tissues repair in the whole body.

The unbalance between the energy storage and energy expenditure conduct to an increased adipose tissues mass leading to obesity-linked type 2 diabetes (T2D) [8,9]. Defined by adipocyte hyperplasia and hypertrophy, obesity leads to the establishment of a cellular "dysfunctional" state in adipocytes and an altered adipokines secretion profile $[10,11]$.

In this study, we hypothesize that excess in energy supply through high-fat diets (HFD) alter the differentiation potential of ASC favoring adipocytes over the endothelial pathway. This will alter the vascular renewal po- 
tential of ASC to the detriment of adipose tissue expansion needed to store energy excess. Thus, significant progress has been made in our understanding of the relation between HFD feeding and adipose tissue dysfunction. Nevertheless, experimental evidence for HFD impact on ASC segregation remains to be elucidated. Therefore, we propose a possible role for HFD-linked metabolic alterations on the ASC differentiating potential. Herein, we evaluated the impact of HFD on the unbalance between the endothelial versus the adipocytes differentiating potential of ASC.

\section{Materials and Methods}

\subsection{Experimental Protocol}

The animal protocol was approved by the Animal Care and Use Committee of the Montreal Heart Institute conforming to the Guide for the Care and Use of Laboratory Animals published by the US National Institutes of Health (NIH Publication No. 85-23, revised 1996). Three groups of $10 \mathrm{C} 57 \mathrm{BL} / 6 \mathrm{~J}$ male mice (Jackson Laboratory, Bar Harbor, MN, USA) were included in this study at 5 weeks of age. Each group was fed either standard diet (SD) used as control ( $6 \%$ fat, $57 \%$ sucrose) or one of the two low cholesterol HFD (34.9\% fat, $26.3 \%$ sucrose) [9]. These latter were iso-caloric but differed in fat nature: VD was composed of soy and cotton oil while AD was composed of lard. Weight gain was monitored during the 20 weeks of protocol and daily food consumption was calculated by subtracting the residual quantity from the supplemented food quantity each day. Energy intake was calculated on the basis of $3.8 \mathrm{kcal} / \mathrm{g}$ for the SD and 5.2 $\mathrm{kcal} / \mathrm{g}$ for both HFD.

\subsection{Biochemical Analysis}

The Intra-Peritoneal Glucose Tolerance Test (IPGTT) was performed after 20 weeks of diet following an overnight fasting. Mice received an intra-peritoneal injection of 20 $\mathrm{g}$ of glucose $/ \mathrm{kg}$ of body weight and glucose level was monitored by tail vein bleeding at $0,15,60$ and 90 minutes post-injection. Area under the curve (AUC) of the glycaemia plotted against the time was used to evaluate mice glucose tolerance. At sacrifice, after an overnight fast (18 hr), blood samples were gathered by cardiac exsanguinations and plasma insulin concentration was measured using the mouse EIA kit (ALPCO).

\subsection{Isolation of ASC}

Mice were anesthetized using Ketamine and Xylazine and euthanized by cardiac exsanguination. Abdominal visceral white adipose tissue was extracted, washed and minced in low glucose Dulbecco's Modified Eagle's
Medium (DMEM), then digested in a $1 \mathrm{mg} / \mathrm{ml}$ type I collagenase solution (GIBCO) for 45 minutes at $37^{\circ} \mathrm{C}$ in a shaking water bath. Afterwards, adipocytes and nonadipose cells were separated by centrifugation and the resulting upper layer (containing the adipose cells) was collected and immediately plated in DMEM [12]. The remaining pellet, containing non-adipose cells, was digested for an additional 20 minutes in a $1 \mathrm{mg} / \mathrm{ml}$ type I collagenase solution in a $37^{\circ} \mathrm{C}$ water bath under constant shaking. Afterwards, cells were diluted in basal medium and filtered through a $100 \mu \mathrm{m}^{2}$ strainer to discard cellular debris. The number of viable cells was determined by Trypan blue. The non-adipose cell density was defined by the ratio of their number per milligram of adipose tissue. These cells, mainly ASC but also including leukocytes and small amount of endothelial cells, were collected and stored in liquid nitrogen in 80\% DMEM, $10 \%$ FBS and $10 \%$ DMSO until analysis. This cell fraction is named ASC.

Prior using the ASC isolated from HFD-fed mice, ASC were isolated from 15-week-old healthy male C57/ BL6J mice and used as control in morphometric and confocal analyses of specific cell markers. These ASC were grown in DMEM in a 12-well plates coated with a rat tail collagen film (Roche Applied Science, IN) according to the manufacturer instruction. Culture medium was changed every 2 - 3 days and pictures were taken using a light microscope (BX45 of Olympus) or stained and analyzed by confocal microscopy.

\subsection{Confocal Microscopy}

Cells were washed with PBS, fixed in $2 \%$ paraformaldehyde for 15 minutes and permeabilized with $0.1 \%$ Triton $\mathrm{X}-100$. Nonspecific binding sites were blocked using a $2 \%$ normal serum solution. After washing, cells were incubated in $1 \%$ normal serum containing the appropriate primary antibody: mesenchymal marker CD44 (BD Biosciences Pharmingen; San Jose, CA), adipogenic lineage cell and stromal progenitor cell markers fatty acid-binding protein-2 (aP2, Abcam; Cambridge, MA) and preadipocyte factor-1 (Pref-1, MBL International Corp.; Woburn, MA), endothelial markers CD31 (SantaCruz Biotechnology; CA) and von Willebrand factor (vWF, Chemicon International; Nepean, Ontario). Cells were then washed and incubated with the corresponding secondary antibody: goat-anti-rabbit Alexa Fluor 488-conjugated (Invitrogen, Carlsbad, CA), goat-anti-rat Alexa Fluor 555conjugated (Invitrogen) or donkey-anti-goat Alexa Fluor 555-conjugated (Invitrogen). Cell nuclei were visualized with Topro-3 iodide (Invitrogen). Slides were analyzed using a confocal microscope (Zeiss LSM 510 63x/1.4 Plan-Apochromat objective, Carl Zeiss Canada, Toronto, Ontario). 


\subsection{Adipogenic Differentiation of ASC}

To assess adipogenic differentiation potential, frozen ASC were thawed and seeded in cell culture flasks (25 $\mathrm{cm}^{2}$ ) with DMEM. After a 2 - 3 rounds of division, cells were transferred to type I collagen-coated 24-well culture plates. When ASC reached $80 \%$ - 90\% confluence, medium was changed to adipogenic inducing differentiation medium containing DMEM high-glucose supplemented with $5 \%$ FBS, $450 \mu \mathrm{M}$ isobutylmethylxanthine, $0.1 \mu \mathrm{M}$ dexamethasone, $2 \mu \mathrm{M}$ insulin and $1 \mu \mathrm{M}$ of 2,4-thiazolidinediones, with media changes every 2 - 3 days [7]. DMEM low-glucose supplemented with 5\% FBS was used as a control to measure the spontaneous adipogenic differentiation. After 21 days, adipogenic differentiation was measured by Oil-Red-O coloration. Cells were then fixed in $10 \%$ formalin for 15 minutes. $60 \%$ isopropanol was then added before drying wells completely. A stock of $0.5 \%$ ORO staining solution was prepared in $60 \%$ triethyl phosphate (TEP, Sigma) and filtered. A working solution was prepared by mixing $30 \mathrm{ml}$ of ORO stock solution with $50 \mathrm{ml}$ of PBS, filtered through a $1 \mu \mathrm{m}$ filter and added to the dried wells. Following a 1 hour incubation, the ORO solution was removed and cells were washed in PBS prior to picture acquisition using a light microscope (BX45 of Olympus) equipped with a video camera (QIMAGING, QICAM, Olympus). The quantification of ORO positive cells was made using the ImagePro program. Red pixels of $4-15$ fields using two wells for each condition (adipogenic or control media) were counted for each mouse. Adipogenic differentiation potential of ASC for each diet was determined by the percentage of the number of red pixels over the total number of pixels. The adipogenic differentiation potential was calculated by comparing the adipogenic and the basal media within a same diet. Data from 4 - 6 mice per group per medium were compiled.

\subsection{Quantification of mRNA Level}

Total RNA was isolated from frozen ASC using Qiazol reagent according to the manufacturer's instructions (Qiagen, Toronto, ON, Canada). Single-strand cDNA was synthesized according to the procedure in the iScript cDNA Synthesis Kit manual (Bio-Rad Laboratories, Montreal, QC, Canada). Q-PCR reactions were carried out using the Brilliant-II SYBR ${ }^{\circledR}$ Green Master-Mix (Stratagene, Mississauga, ON, Canada) and specific primers for:

- aP2: forward primer 5'-TGG GAA CCT GGA AGC TTG TCT C-3' reverse primer 5'-GCT GAT GAT CAT GTT GGG CTT G-3';

- PPAR $\gamma 2$ forward primer 5'-AGC ATG GTG CCT TCG CTG AT-3' reverse primer 5'-GGT GGA GAT GCA GGT TCT AC-3';

- $\mathrm{C} / \mathrm{EBP} \alpha$ forward primer 5'-GAG CCG AGA TAA
AGC CAA ACA-3' reverse primer 5'-CGG TCA TTG TCA CTG GTC AAC T-3';

- TNF $\alpha$ forward primer 5'-CAT-CTT-CTC-AAAATT-CGA-GTG-ACA-A-3' reverse primer 5'-TGGGAG-TAG-ACA-AGG-TAC-AAC-CC-3';

- Cyclin B1: forward primer 5'-TGG CCT CAC AAA GCA CAT GA-3' reverse primer 5'-GCT GTG CCA GCG TGC TAA TC-3'.

The mRNA levels were normalized to Cyclophilin-A expression levels (Fwd 5'-CCG-ATG-ACG-AGC-CCTTGG-3'; Rev 5'-GCC-GCC-AGT-GCC-ATT-ATG-3'). The targeted and referenced genes were amplified in duplicate in the same run using the Mx3000P Q-PCR System (Stratagene).

The relative quantification of target genes was determined using the MxProTM Q-PCR software version 3.00 (Strategene). Briefly, $\mathrm{Ct}$ average of each duplicate was calculated for each gene and Cyclophilin-A and the $\triangle \mathrm{CT}$ (CTgene-CTCyclo-A) was determined. The control adipose tissue sample was chosen as a reference sample and set as $100 \%$ of gene quantity. Finally, the mRNA abundance of other samples to the mRNA abundance of the control adipose tissue was calculated with use of the formula $2^{-\Delta \Delta \mathrm{CT}}$.

\subsection{Endothelial Differentiation of ASC}

Frozen ASC were thawed and amplified 2 - 3 times. Cells were then plated on type 1 collagen-coated Lab-Tek chamber slides (Thermo Fisher Scientific, NY). When ASC reached $80 \%$ - 90\% confluence, medium was changed for endothelial differentiation medium, consisting of endothelial growth medium-2 (EGM-2, Lonza, Walkersvelle, MD) supplemented with 5\% FBS, vascular endothelial growth factor $(50 \mathrm{ng} / \mathrm{ml}$, Peprotech, Rocky Hill, NJ) and epithelial growth factor (10 ng/ml, Peprotech, Rocky Hill, NJ) [13,14]. DMEM supplemented with 5\% FBS was used as a reference control medium to evaluate basal endothelial cell content. After 0,7 or 14 days, endothelial differentiation was evaluated through the acquisition of endothelial markers detected by confocal microscopy. Staining procedure was performed as described above.

\subsection{Immunohistologic Analysis of CD36 Expression in Fat Tissues}

To evaluate macrophage infiltration, white adipose tissue was fixed in $10 \%$ formalin, embedded in parafilm and cut in $4 \mu \mathrm{m}$ sections. White adipose tissue sections were analyzed by immunohistology using the following a rabbit polyclonal anti-CD36 (Santa Cruz sc-9154, $200 \mathrm{ug} / \mathrm{ml}$ ) diluted: $1 / 100$ and biotinylated anti-rabbit IgG (Vector, BA-1000, $1.5 \mathrm{mg} / \mathrm{ml}$ ) diluted $1 / 400$. Slides were then stained for 30 min using an avidin-biotin complex (Vec- 
tor Labs) followed by a DAB-peroxidase revelation. Finally, the sections were counterstained with hematoxylin (Leica Biosystems, Richmond, USA) and mounted with permount (Fisher Scientific, Fair Lawn, USA). For each tissue, three images were acquired (Olympus) and analyzed for nucleated CD36 positive cells using segmentation with image Pro 6.2 (Mediacybernetics, Bethesda, USA).

\subsection{Statistical Analysis}

All results are presented as mean \pm SEM. Repeated measure analysis of covariance (ANCOVA) was used to compare differences in weight gain divided by initial weight and a one-way analysis of variance (ANOVA) model was used to study the glycemic parameters. Nonparametric ANOVA (Kruskal-Wallis test) followed by a Dunn test were used to compare non-adipose cell number in adipose tissue ( $\mathrm{n}=9 \mathrm{mice} / \mathrm{diet})$. Non-parametric ANOVA (Kruskal-Wallis test) followed by a Mann-Whitney test were used to compare the adipogenic differentiation potential within a same diet and by a Dunn test to compare the three diets for a same culture media. ANOVA for multiple testing followed by a Tukey test was used to compare mRNA level of different genes. Analysis of CD36 density was made as follows (by a statistician of the Montreal Heart Institute Coordinating Center (MHI$\mathrm{CC})$ ): Areas were compared between diets using an analysis of variance (ANOVA) with heterogeneous (unequal) variances. Measurement repetitions for each animal were considered as independent observations. Probability values were considered significant at $P<0.05$.

\section{Results}

\subsection{Metabolic Parameters Influenced by the Fat Origin in HFD}

The extent of metabolic alterations was measured 20 weeks after the beginning of the protocol [9]. Obesity was developed in both HFD groups, as demonstrated by a $150 \%$ weight gain for VD and $135 \%$ for AD measured up to to their initial weight, compared to SD group (64\% increased weight). In addition, AUC values show overt glucose intolerance in VD $(45.5 \pm 3.1)$ and AD (44.6 \pm 1.0$)$ groups compared to SD group $(28.6 \pm 1.1)$. Metabolic alteration was also reflected by a high fasting glycaemia in $\operatorname{VD}(9.00 \pm 0.57 \mathrm{mmol} / \mathrm{L})$ and $\mathrm{AD}(7.73 \pm 0.47 \mathrm{mmol} / \mathrm{L})$ groups compared to control $(3.97 \pm 0.20 \mathrm{mmol} / \mathrm{L})$. All together these data delineate a T2D state in HFD fed mice coupled to hyperinsulinemia in the AD group [9].

\subsection{Characteristics of Adipose Tissue from Mice Fed with the Three Diets}

In order to evaluate the impact of diet on abdominal white adipose tissue cell contents and aspects were characterized. Mature adipocytes were hypertrophic and hyperplasic in HFD groups compared to control $[9,12]$. However, the number of non-adipose cells isolated per mg of adipose tissue was comparable in all mice group (Figure 1(a)). Moreover, the immunohistologic analysis of the CD36 expression in the white adipose tissues revealed a $4-7$ fold increase in density of nucleated $\mathrm{CD}^{+} 6^{+}$cells in the VD and the AD groups, compared to SD group, showing a higher macrophage infiltration in the adipose tissue of the corresponding mice (Figure 1(b)).

\subsection{Morphology and Protein Expression of ASC}

Prior to evaluating the differentiation potential of ASC from mice fed with the 3 diets, we verified whether ASC retained their cellular characteristics after freezing and after seeding on collagen matrix. Two cryopreservation agents, DMSO and PVP, were tested. The rate of ASC survival was 2-fold higher post-DMSO cryopreservation compared to the PVP medium. The first represented an accelerated growth kinetic during the first week in culture (data not shown). Based on these results, all isolated

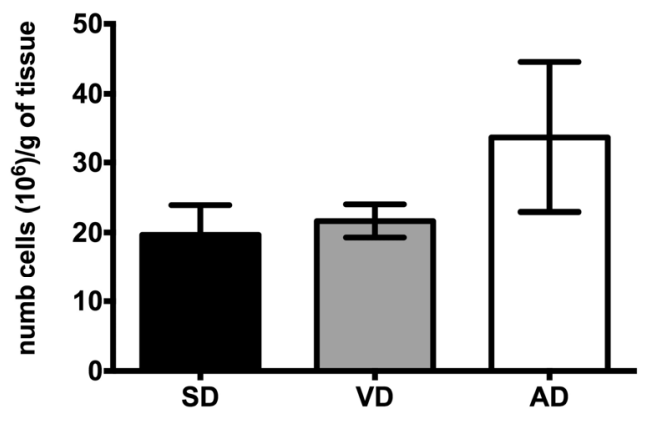

(a)

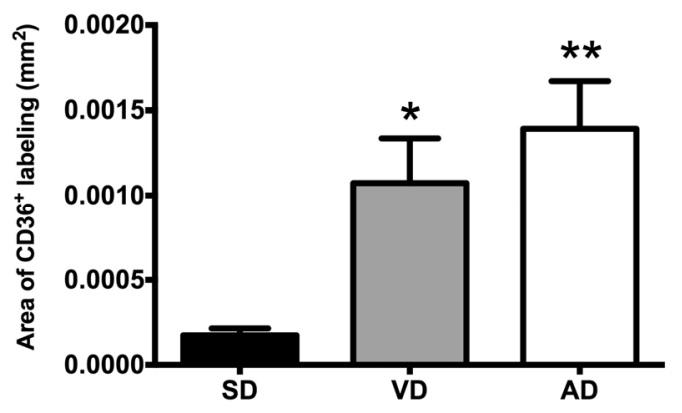

(b)

Figure 1. Cellular characteristics of adipose tissues of SD, VD and AD fed mice. Non-adipose cellular density (a) and area of $\mathrm{CD}^{+} 6^{+}$nucleated cells (b) were measured by cell count and immunohistology analysis respectively. Immunohistologic analysis of the CD36 density was evaluated at 20X. $n=9$ for cellular density. $n=4$ for the SD, $n=5$ for the VD and $n=8$ for the AD for cell area. $* P<0.01, * * P<0.001$ vs SD. 
ASC were cryopreserved in DMSO until usage.

During the amplification phase, the majority of ASC at 4, 6 and 12 days post-seeding had a fibroblastic-like morphology characteristic (Figure 2A). Further, about 95\% of cells expressed the mesenchymal stromal marker CD44 and a high level of the pre-adipocyte marker Pref- 1 while maintaining the expression of aP2 indicating an adipogenic potential (Figure 2A). Most of the cells aggregate in colonies (Additional Figure S1). The elevated expression of CD44, Pref-1 and aP2 and the presence of colonies suggest that once seeded on collagen, ASC cryopreserved in DMSO maintain their adipogenic characteristics.

\subsection{HFD Modulate the Adipogenic Differentiation Potential of ASC}

ASC adipogenic potential was evaluated through the mRNA expression levels of key genes involved in the process: aP2, FAS, PPAR $\gamma 2$ and $\mathrm{C} / \mathrm{EBP} \alpha . \operatorname{PPAR} \gamma 2$ mRNA levels tend to decrease in the VD group, but only $\mathrm{C} / \mathrm{EBP} \alpha$ expression level was significantly reduced in this group compared to control (Figure 3). For its part, $\mathrm{AD}$ decreases PPAR $\gamma 2$ and $\mathrm{C} / \mathrm{EBP} \alpha$ mRNA levels by approximately by up to 4 folds compared to SD and VD groups. The difference between these 2 HFD groups was observed in decrease aP2 mRNA expression level and increased FAS mRNA expression level in AD group (Figure 3).
Since the proliferation potential of ASC has been negatively correlated to their differentiation capacity in obese [15], we measured the mRNA expression level of mitotic cyclin B1. Surprisingly, in ex vivo ASC, cyclin B1 mRNA level was 3-fold higher in the VD group $(228.50 \pm 1.77, P<0.001)$ compared to the AD group $(76.30 \pm 7.74)$ and the SD group $(76.25 \pm 7.28)$ (Figure 3(e)).

To further characterize the adipogenic potential, ORO experiments were performed on ASC. After 21 days of culture in adipogenic medium, adipocyte differentiation of ASC tends to increase in the three groups compared with their corresponding control media (Figure 3(f)). The adipogenic differentiation of ASC was greater for the two HFD groups compared to the SD group. Indeed, when control and adipogenic conditions were compared, a 4.3 and 3.0 fold increase in ORO positive area was calculated for the $\operatorname{VD}(P=0.0028)$ and the $\operatorname{AD}(P=$ $0.0001)$ respectively, while only a 1.5 fold induction was seen for the SD group. This enhanced adipogenic potential of ASC of HFD groups was also reflected in an higher level of FAS mRNA expression level significant in the case of AD group $(P<0.05)$ (Figure 3(b)).

\subsection{Vegetal HFD Abrogates the Endothelial Differentiation of ASC}

Response of ASC to endothelial differentiation medium was clearly distinct between the 3 groups of mice. Cells

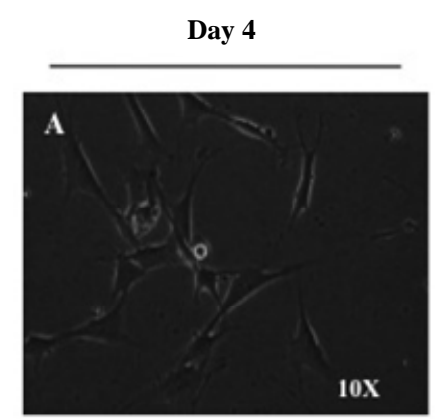

CD 44

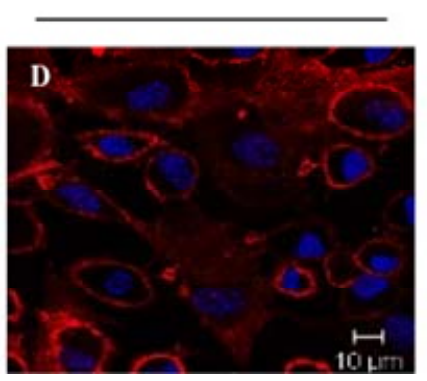

Day 6

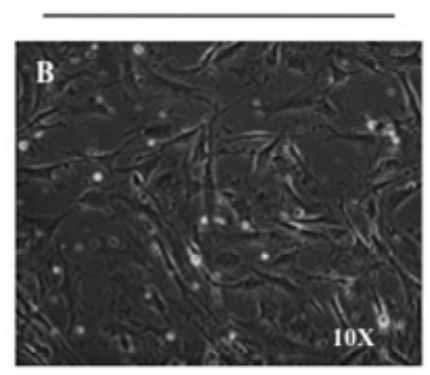

Pref-1

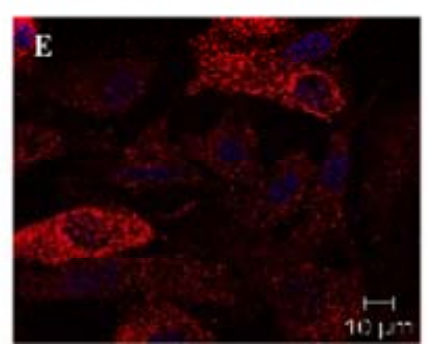

Day 12

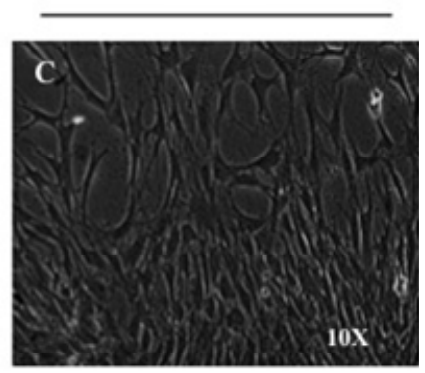

aP2

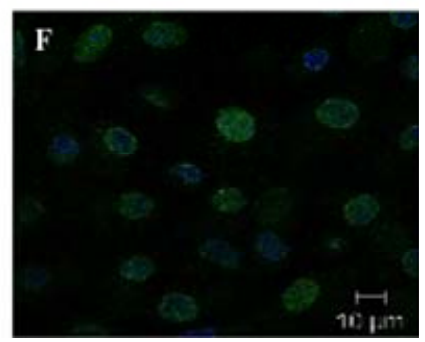

Figure 2. ASC morphology and proteins expression on collagen matrix. ASC were grown on collagen matrix. Cell morphology was evaluated by observation at 4, 6 and 12 days post cell seeding. Expression of markers for mesenchymal stromal cells $\left(\mathrm{CD}_{4}^{+}\right)$, stromal progenitor cells $\left(\mathrm{Pref}_{-1} \mathrm{1}^{+}\right)$and adipocytes lineage cells $\left(\mathrm{aP2}^{+}\right)$were evaluated by confocal microscopy $(63 \mathrm{X})$ after immunostaining. 


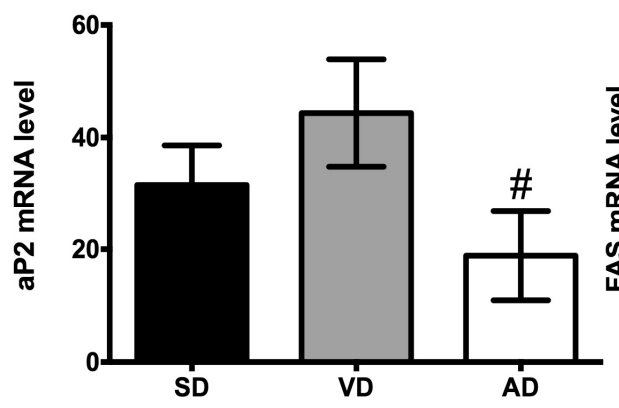

(a)

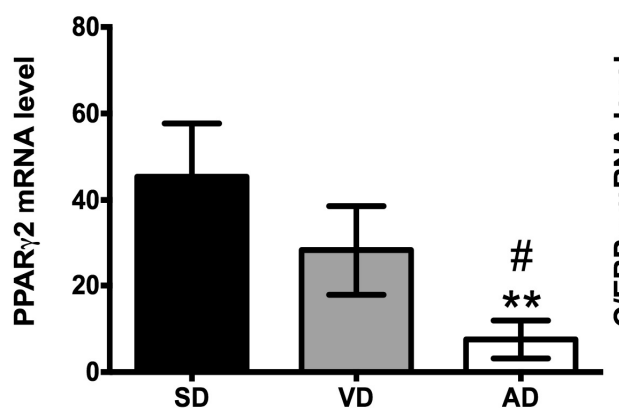

(c)

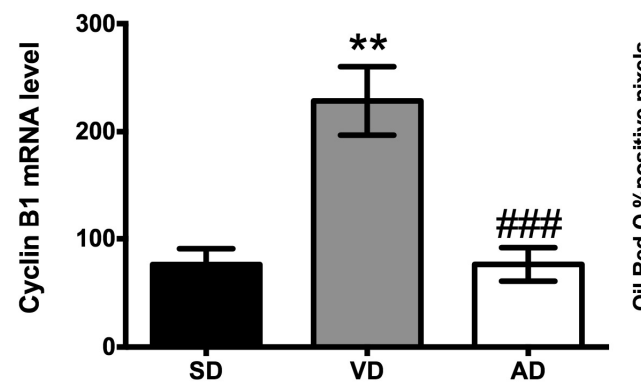

(e)

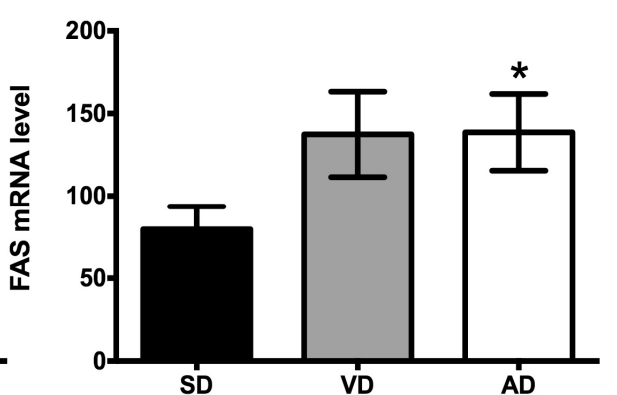

(b)

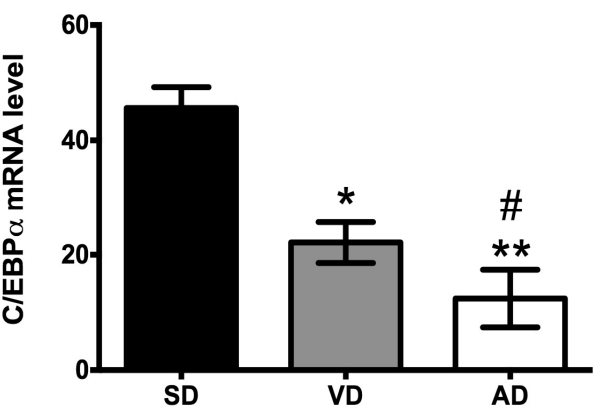

(d)

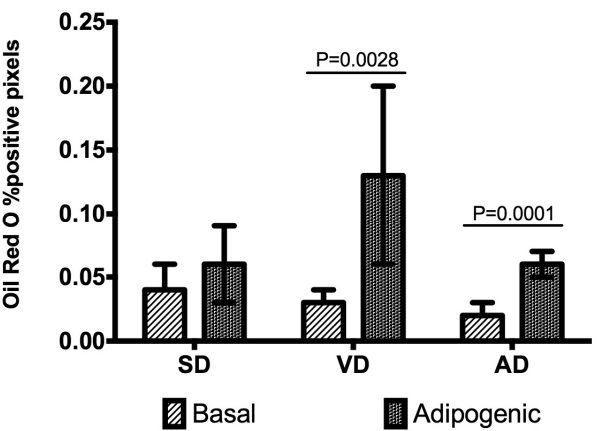

(f)

Figure 3. Quantification of mRNA level of adipogenic genes in SD $(n=4)$, VD $(n=4)$ and AD (n = 3) groups. aP2 (a), FAS (b), PPARg (c), C/EBP $\alpha$ (d) and cyclin B1 mRNA levels were evaluated by Q-PCR. Specific gene expression in uncultured ASC was normalized to reported gene cyclophiline-A. Each sample was tested in duplicate. $* P<0.05, * * P<0.01$ vs SD; \#P<0.05, $\# \# \#>0.001$ vs VD. (f) The adipogenic differentiation potential, expressed as a percentage of colored pixels (i.e. ORO positive staining) over total pixel number, was measured for ASC after 21 days of culture media with or without adipogenic agent.

originating from the SD group rapidly acquired vWF expression after 7 days and subsequently declined (Figures 4A-C). In the AD group, differentiation of vWF cells was successful but required an additional week in endothelial medium (Figures 4D and E). In contrast, ASC from the VD group were persistently negative for vWF cells (Figures 4F and G). Thus, we demonstrated a delayed endothelial differentiation process with ASC from the AD group compared to the SD group while this process was completely inhibited in VD group. To be certain that the negative result of the VD group was not due to a specific defect in $\mathrm{vWF}$ expression, the mRNA expression of CD31, another endothelial cell marker was evaluated. Following 7 days in endothelial differentiation medium,
CD31 cells were observed for the SD group whereas ASC from the VD group were negative for this marker (Figures $4 \mathbf{H}$ and I) (Note that the CD31 cytosolic staining in our assays is due to the fixation step of the staining protocol, which results in the permeabilisation of the cell membrane). These results confirmed the incapacity of ASC from the VD group to differentiate into endothelial cells.

\section{Discussion}

While many studies have focused on the effects of metabolic abnormalities on adipocyte activity and resulting systemic implications, consequences for ASC survival, amplification and differentiation potential have not 

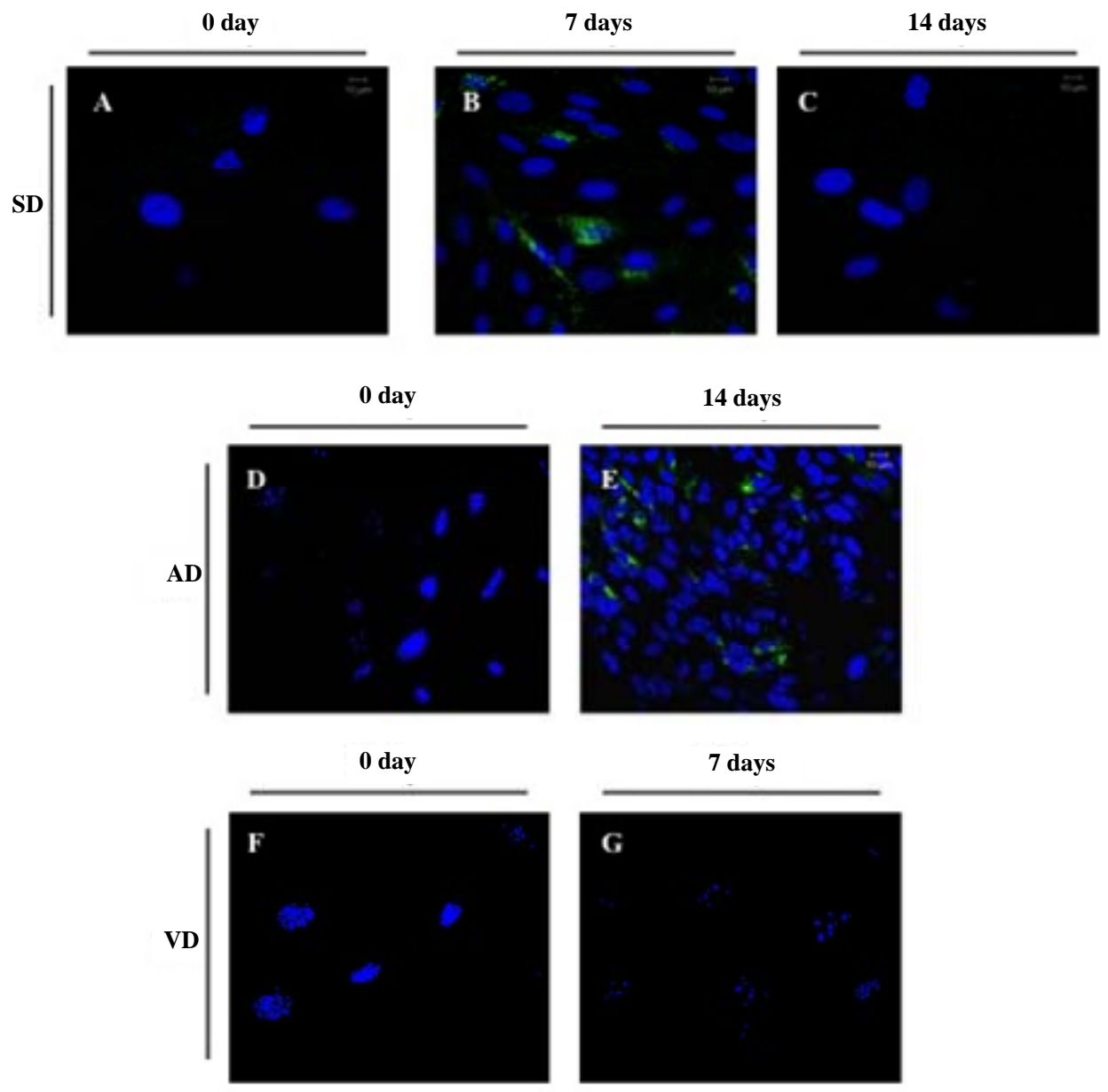

SD
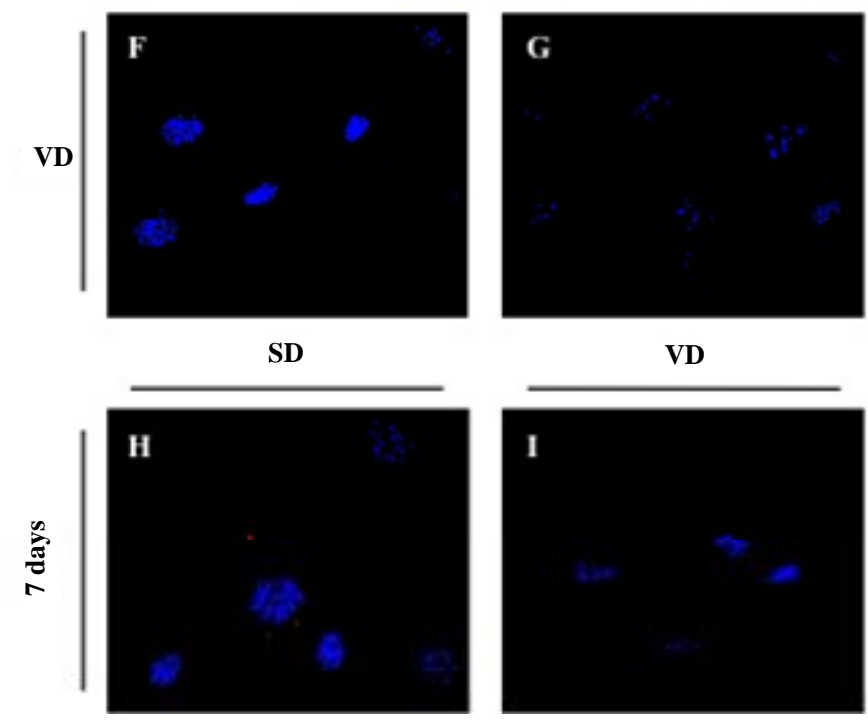

VD

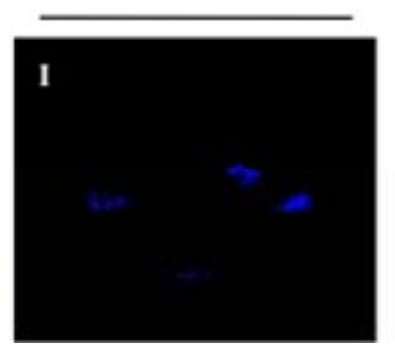

Figure 4. Evaluation of the ASC endothelial differentiation potential. Expression of the endothelial cell marker vWF (green label) in ASC from the SD group after 0 (A), 7 (B) and 14 (C) days, from the VD group after 0 (D) and 7 (E), and from the AD group after 0 (F) and 14 (G) days in endothelial media. Expression of the endothelial cell marker CD31 ${ }^{+}$(red label) in ASC cultures from SD (H) and VD (I) groups analyzed by confocal microscopy after 7 days in endothelial media.

been fully addressed. This question is of clinical importance, white fat tissues are being considered as an attractive source of autologous stem cells in regenerative medicine.

The identification of adult tissues as a source of stem cells has opened tremendous possibilities in regenerative medicine. Early clinical studies using bone marrow-derived stem cells have provided promising results and demonstrate their therapeutic potential. However, important challenges, including the cell harvesting procedure from the bone marrow, may limit their utilization [16]. Other studies have demonstrated the in vitro and in vivo angiogenic and tissue regeneration potential of ASC representing an attractive alternative to overcome this clinical limitation [7,17,18]. ASC, a promising new source of adult stem cells for cardiac repair and bone regeneration, were shown to give rise to both adipocytes and endothelial cells [7,19]. Knowing the major impact of metabolic status on adipose tissue and inflammatory infiltrate [10], we hypothesized that the balance between adipogenic 
and endothelial differentiation processes of ASC is altered in a context of metabolic disorders. To evaluate this hypothesis, we used two different HFDs that we show induced T2D in male mice [20].

In our experimental setting, the HFD with lipids from vegetal source (VD) induced a T2D status characterized by obesity-linked fasting hyperglycemia and glucose intolerance but in contrast to the $\mathrm{AD}$ no hyperinsulinemia. At the cellular level, adipocyte hypertrophy was exacerbated under $\mathrm{AD}$, compared to the VD group. This result can be explained by hyperinsulinemia state of AD-fed mice. Indeed, it has been demonstrated that high circulating level of insulin and insulin sensitivity are associated with adipocyte hypertrophy [21]. When exposed to a low adipogenic medium, ASC from VD and AD but not from the SD group demonstrated a significant adipogenic differentiation potential when compared to the basal condition. The use of a low adipogenic medium in our study could explain the non-significant adipogenic differentiation for the SD group (compared to its own control), contrary to the VD and the $\mathrm{AD}$ groups (compared to their respective control). The microenvironment from which originate ASC could have shape the capacity of ASC to respond to exogenous adipogenic stimuli provided by pro-adipogenic differentiation medium in vitro. This enhance adipogenic potential could be linked to different factors. Adipose tissue expansion during obesity development is associated with a chronic low-grade inflammatory cell infiltration that is part of the non-adipose cell pool [22]. CD36 is expressed mainly by infiltrating nucleated macrophages and was found to be enhanced at a comparable level in abdominal white fat from the two HFD mice groups. This suggests that for a similar number of non-adipose cells, a lower proportion of preadipocytes is expected to be present in VD and AD cell fractions when compared to SD containing a much lower count of macrophages. In this case, other factors than the available number of ASC certainly impact on the adipogenic potential of cells isolated from the VD and AD-fed mice.

Cellular response to pro-adipogenic stimulation could be determined by their basal expression of lipogenic genes. PPAR $\gamma$ and aP2 mRNA are typically up-regulated during the differentiation process of preadipocytes into adipocytes while $\mathrm{C} / \mathrm{EBP} \alpha$ was also linked to insulin sensitivity [23]. However, a decreased level of these mRNA was associated with metabolic diseases such as T2D and to a decrease differentiation of newly formed adipocytes $[24,25]$. In agreement with this, AD-fed mice had more advanced T2D with hyperinsulinemia when compared to VD-fed mice and had the lowest expression level of lipogenic genes even if cells were exposed in vivo to high concentration of free fatty acids.

The activation status of macrophages, primary source of TNF $\alpha$ was reported to contribute in a major way to shape the adipogenic potential of fat tissue [26]. Macrophages are more strongly induced in the adipose tissue in the abdominal white fat of the AD than in the VD group reflected by a 2.5 -fold increase at the mRNA level of $\mathrm{TNF} \alpha$ in corresponding adipocytes [9]. The lower adipogenic genes expression levels in ASC from AD could then also be explained by their exposition to a higher proportion of activated macrophages in fat tissue, producing TNF $\alpha$ which is reported to inhibit PPAR $\gamma$ expression $[27,28]$. This reduction was confirmed by our QPCR analyses on fat tissues [20]. As TNF $\alpha$ was reported to promote preadipocyte proliferation, we could have expected an increased cyclin B1 mRNA level in the AD compared to the VD ASC. Conversely, high levels of $\mathrm{TNF} \alpha$ such as in $\mathrm{AD}$ fat tissue, is also associated with the induction of insulin resistance, stimulation of lipolysis and preadipocyte apoptosis, and contribute to the reduction of the number of cycling cells [29]. Nevertheless, the expression level of lipogenic genes could be difficult to extrapolate into adipogenic potential as demonstrated by the ASC from SD that had the highest expression levels but only a marginal response to the adipogenic media.

With prolonged exposure to high free fatty acid concentrations, the induced white fat tissue expansion implies the development of neo-vessels to provide nutrients [30]. ASC have the capacity to differentiate into endothelial cells, a characteristic that arouses the interest in these cells for vascular regenerative medicine. The nature and stiffness of the extracellular matrix proteins used for ASC cultures influences their adhesion, proliferation and differentiation but are not affected by the cryopreservation process [31-36]. Detection of cells with an endothelial phenotype and markers in our cultures initiated with ASC from the SD and the AD indicates that the cryopreservation, culture condition, selected collagen matrix and selected endothelial differentiation medium were appropriate to maintain the endothelial differentiation potential of ASC. However, hyperglycemia has been reported to impair bone marrow derived endothelial progenitor cells functions including endothelial differentiation [37-39]. ASC isolated from the abdominal visceral white fat of the HFD groups had an elevated fasting glycemia, demonstrated an absence of endothelial differentiation for the VD group while it was delayed, but still effective, for the AD group. As both HFD groups were diabetic, other important factors can negatively impact endothelial differentiation capacities of ASC. The hyperinsulinemic status of $\mathrm{AD}$ mice, not found in VD mice, could have partially protected the ASC potential to differentiate into EC, explaining the 1-week delay to detect vWF expression. Indeed, insertion of insulin implants prior to arterial catheter injury was demonstrated to increase the number of circulating progenitor cells and the reendothelialization 
in the rodent model $[40,41]$.

ASC derived from VD-fed mice were unable to give rise to vWF or CD31 positive cells. Linoleic acid (omega6) content was the same between the two HFD, but the percentage of palmitic acid was lower in the VD than in the $\mathrm{AD}$, excluding this track as a possible explanation for this defect. In contrast, the $\mathrm{AD}$ has two times more $\alpha$ linolenic acid, an omega-3 fatty acid, than the VD. A higher ratio of omega-3 over omega- 6 fatty acid was associated with a higher level of later stage progenitor cells [42]. Thus, the lower ratio of omega-3 over omega- 6 in the VD could explain the abrogated endothelial cell differentiation in this diet. The enhanced adipogenic potential and defective endothelial differentiation of ASC from the VD group suggests that a ratio of unsaturated fatty acids may contribute to shaping the equilibrium between differentiation pathways.

Taken together, our results underline the contribution of HFD to induce metabolic alterations modulating the proliferation and the balance between the adipogenic and the endothelial differentiation process of progenitor cells in adipose tissue. Furthermore, we have demonstrated that these cellular processes are differently modulated by the lipid composition of the diet. These results highlight the importance of evaluating the ASC differentiation behavior in a context of autologous cell-based therapy for the repair of vascular tissues in obese and diabetic donors.

\section{Acknowledgements}

The authors wish to acknowledge Louis Villeneuve from the confocal microscopy service of the Montreal Heart Institute, Marie-Élaine Clavet-Lanthier and Dominique Lauzier from the histopathology service for their help in the Oil-Red-O staining procedures and Sylvie Lévesque from the Montreal Heart Institute Coordinating Center for the statistical analyses.

\section{REFERENCES}

[1] S. Galic, J. S. Oakhill and G. R. Steinberg, "Adipose Tissue as an Endocrine Organ," Molecular and Cellular Endocrinology, Vol. 316, No. 2, 2010, pp. 129-139. doi:10.1016/j.mce.2009.08.018

[2] M. Pittenger, P. Vanguri, D. Simonetti and R. Young, "Adult Mesenchymal Stem Cells: Potential for Muscle and Tendon Regeneration and Use in Gene Therapy," Journal of Musculoskeletal and Neuronal Interactions, Vol. 2, 2002, pp. 309-320.

[3] K. W. Park, D. S. Halperin and P. Tontonoz, "Before They Were Fat: Adipocyte Progenitors," Cell Metabolism, Vol. 8, No. 6, 2008, pp. 454-457. doi:10.1016/i.cmet.2008.11.001

[4] Q. Q. Tang, T. C. Otto and M. D. Lane, "Commitment of C3H10T1/2 Pluripotent Stem Cells to the Adipocyte Lin- eage," Proceedings of the National Academy of Sciences of the United States of America, Vol. 101, No. 26, 2004, pp. 9607-9611. doi:10.1073/pnas.0403100101

[5] F. M. Gregoire, C. M. Smas and H. S. Sul, "Understanding Adipocyte Differentiation," Physiological Reviews, Vol. 78, No. 3, 1998, pp. 783-809.

[6] P. Zhang, N. Moudgill, E. Hager, et al., "Endothelial Differentiation of Adipose-Derived Stem Cells from Elderly Patients with Cardiovascular Disease," Stem Cells and Development, Vol. 20, No. 6, 2011, pp. 977-988. doi:10.1089/scd.2010.0152

[7] Y. Cao, Z. Sun, L. Liao, Y. Meng, Q. Han and R. C. Zhao, "Human Adipose Tissue-Derived Stem Cells Differentiate into Endothelial Cells in Vitro and Improve Postnatal Neovascularization in Vivo," Biochemical and Biophysical Research Communications, Vol. 332, No. 2, 2005, pp. 370-379. doi:10.1016/j.bbrc.2005.04.135

[8] P. Singla, A. Bardoloi and A. A. Parkash, "Metabolic Effects of Obesity: A Review," World Journal of Diabetes, Vol. 1, No. 3, 2010, pp. 76-88. doi:10.4239/wjd.v1.i3.76

[9] S. El Akoum, V. Lamontagne, I. Cloutier and J. F. Tanguay, "Nature of Fatty Acids in High Fat Diets Differentially Delineates Obesity-Linked Metabolic Syndrome Components in Male and Female C57BL/6J Mice," Diabetology \& Metabolic Syndrome, Vol. 3, No. 1, 2011, p. 34.

[10] N. Ouchi, J. L. Parker, J. J. Lugus and K. Walsh, “Adipokines in Inflammation and Metabolic Disease," Nature Reviews Immunology, Vol. 11, No. 2, 2011, pp. 85-97. doi:10.1038/nri2921

[11] V. DeClercq, C. Taylor and P. Zahradka, "Adipose Tissue: The Link between Obesity and Cardiovascular Disease," Cardiovascular \& Hematological Disorders Drug Targets, Vol. 8, No. 3, 2008, pp. 228-237. doi: $10.2174 / 187152908785849080$

[12] S. El Akoum, I. Cloutier and J. F. Tanguay, "Vascular Smooth Muscle Cell Alterations Triggered by Mice Adipocytes: Role of High-Fat Diet," Journal of Atherosclerosis and Thrombosis, Vol. 19, 2012, pp. 1128-1141. doi: $10.5551 /$ jat. 13482

[13] L. S. Ferreira, S. Gerecht, H. F. Shieh, et al., "Vascular Progenitor Cells Isolated from Human Embryonic Stem Cells Give Rise to Endothelial and Smooth Muscle Like Cells and Form Vascular Networks in Vivo," Circulation Research, Vol. 101, 2007, pp. 286-294. doi:10.1161/CIRCRESAHA.107.150201

[14] W. Jianguo, L. Tianhang, Z. Hong, et al., "Optimization of Culture Conditions for Endothelial Progenitor Cells from Porcine Bone Marrow in Vitro," Cell Proliferation, Vol. 43, No. 4, 2010, pp. 418-426. doi:10.1111/j.1365-2184.2010.00688.x

[15] V. Van Harmelen, K. Rohrig and H. Hauner, "Comparison of Proliferation and Differentiation Capacity of $\mathrm{Hu}-$ man Adipocyte Precursor Cells from the Omental and Subcutaneous Adipose Tissue Depot of Obese Subjects," Metabolism: Clinical and Experimental, Vol. 53, No. 5, 2004, pp. 632-637. doi:10.1016/j.metabol.2003.11.012

[16] S. Siddiq, D. Pamphilon, S. Brunskill, C. Doree, C. Hyde 
and S. Stanworth, "Bone Marrow Harvest versus Peripheral Stem Cell Collection for Haemopoietic Stem Cell Donation in Healthy Donors," Cochrane Database of Systematic Reviews, 2009, Article ID: CD006406. doi:10.1002/14651858.CD006406.pub2

[17] A. Miranville, C. Heeschen, C. Sengenes, C. A. Curat, R. Busse and A. Bouloumie, "Improvement of Postnatal Neovascularization by Human Adipose Tissue-Derived Stem Cells," Circulation, Vol. 110, No. 3, 2004, pp. 349-355. doi:10.1161/01.CIR.0000135466.16823.D0

[18] L. Cai, B. H. Johnstone, T. G. Cook, et al., "IFATS Collection: Human Adipose Tissue-Derived Stem Cells Induce Angiogenesis and Nerve Sprouting Following Myocardial Infarction, in Conjunction with Potent Preservation of Cardiac Function," Stem Cells, Vol. 27, No. 1, 2009, pp. 230-237. doi:10.1634/stemcells.2008-0273

[19] V. Planat-Benard, J. S. Silvestre, B. Cousin, et al., "Plasticity of Human Adipose Lineage Cells toward Endothelial Cells: Physiological and Therapeutic Perspectives," Circulation, Vol. 109, No. 5, 2004, pp. 656-663. doi:10.1161/01.CIR.0000114522.38265.61

[20] S. El Akoum, V. Lamontagne, I. Cloutier and J. F. Tanguay, "Nature of Fatty Acids in High Fat Diets Differentially Delineates Obesity-Linked Metabolic Syndrome Components in Male and Female C57BL/6J Mice," Diabetology \& Metabolic Syndrome, Vol. 3, No. 1, 2011, p. 34.

[21] E. Arner, P. O. Westermark, K. L. Spalding, et al., "Adipocyte Turnover: Relevance to Human Adipose Tissue morphology," Diabetes, Vol. 59, No. 1, 2010, pp. 105109. doi:10.2337/db09-0942

[22] S. P. Weisberg, D. McCann, M. Desai, M. Rosenbaum, R. L. Leibel and A. W. Ferrante Jr., "Obesity Is Associated with Macrophage Accumulation in Adipose Tissue," The Journal of clinical investigation, Vol. 112, No. 12, 2003, pp. 1796-1808.

[23] Z. Wu, E. D. Rosen, R. Brun, et al., "Cross-Regulation of C/EBP Alpha and PPAR Gamma Controls the Transcriptional Pathway of Adipogenesis and Insulin Sensitivity," Molecular Cell, Vol. 3, No. 2, 1999, pp. 151-158. doi:10.1016/S1097-2765(00)80306-8

[24] S. T. Nadler, J. P. Stoehr, K. L. Schueler, G. Tanimoto, B. S. Yandell and A. D. Attie, "The Expression of Adipogenic Genes Is Decreased in Obesity and Diabetes Mellitus," Proceedings of the National Academy of Sciences of the United States of America, Vol. 97, No. 21, 2000, pp. 11371-11376. doi:10.1073/pnas.97.21.11371

[25] S. G. Dubois, L. K. Heilbronn, S. R. Smith, J. B. Albu, D. E. Kelley and E. Ravussin, "Decreased Expression of Adipogenic Genes in Obese Subjects with Type 2 Diabetes," Obesity, Vol. 14, No. 9, 2006, pp. 1543-1552. doi:10.1038/oby.2006.178

[26] S. P. Weisberg, D. McCann, M. Desai, M. Rosenbaum, R. L. Leibel and A. W. Ferrante Jr., "Obesity Is Associated with Macrophage Accumulation in Adipose Tissue," Journal of Clinical Investigation, Vol. 112, No. 12, 2003, pp. 1796-1808.

[27] G. J. Tesz, A. Guilherme, K. V. Guntur, et al., "Tumor Necrosis Factor Alpha (TNFalpha) Stimulates Map4k4
Expression through TNFalpha Receptor 1 Signaling to c-Jun and Activating Transcription Factor 2," The Journal of Biological Chemistry, Vol. 282, 2007, pp. 1930219312. doi:10.1074/jbc.M700665200

[28] X. Tang, A. Guilherme, A. Chakladar, et al., "An RNA Interference-Based Screen Identifies MAP4K4/NIK as a Negative Regulator of PPARgamma, Adipogenesis, and Insulin-Responsive Hexose Transport," Proceedings of the National Academy of Sciences of the United States of America, Vol. 103, No. 7, 2006, pp. 2087-2092. doi:10.1073/pnas.0507660103

[29] J. B. Prins, C. U. Niesler, C. M. Winterford, et al., "Tumor Necrosis Factor-Alpha Induces Apoptosis of Human Adipose Cells," Diabetes, Vol. 46, No. 12, 1997, pp. 1939 1944. doi:10.2337/diabetes.46.12.1939

[30] S. Nishimura, I. Manabe, M. Nagasaki, et al., "Adipogenesis in Obesity Requires Close Interplay between Differentiating Adipocytes, Stromal Cells, and Blood Vessels," Diabetes, Vol. 56, No. 12, 2007, pp. 1517-1526. doi:10.2337/db06-1749

[31] A. G. Cristancho and M. A. Lazar, "Forming Functional Fat: A Growing Understanding of Adipocyte Differentiation," Nature Reviews Molecular Cell Biology, Vol. 12, No. 11, 2011, pp. 722-734. doi:10.1038/nrm3198

[32] K. C. O'Connor, H. Song, N. Rosenzweig and D. A. Jansen, "Extracellular Matrix Substrata Alter Adipocyte Yield and Lipogenesis in Primary Cultures of Stromal-Vascular Cells from Human Adipose," Biotechnology Letters, Vol. 25, No. 23, 2003, pp. 1967-1972. doi:10.1023/B:BILE.0000004386.08923.ab

[33] Y. Itoi, M. Takatori, H. Hyakusoku and H. Mizuno, "Comparison of Readily Available Scaffolds for Adipose Tissue Engineering Using Adipose-Derived Stem Cells," Journal of Plastic, Reconstructive \& Aesthetic Surgery: JPRAS, Vol. 63, 2010, pp. 858-864. doi:10.1016/j.bjps.2009.01.069

[34] B. C. Goh, S. Thirumala, G. Kilroy, R. V. Devireddy and J. M. Gimble, "Cryopreservation Characteristics of Adipose-Derived Stem Cells: Maintenance of Differentiation Potential and Viability," Journal of Tissue Engineering and Regenerative Medicine, Vol. 1, No. 4, 2007, pp. 322324. doi:10.1002/term.35

[35] Z. G. Zhao, W. M. Li, Z. C. Chen, Y. You and P. Zou, "Hematopoiesis Capacity, Immunomodulatory Effect and ex Vivo Expansion Potential of Mesenchymal Stem Cells Are not Impaired by Cryopreservation," Cancer Investigation, Vol. 26, 2008, pp. 391-400. doi:10.1080/07357900701788049

[36] J. E. Lee, I. Kim and M. Kim, “Adipogenic Differentiation of Human Adipose Tissue-Derived Stem Cells Obtained from Cryopreserved Adipose Aspirates," Dermatologic Surgery: Official Publication for American Society for Dermatologic Surgery, Vol. 36, No. 7, 2010, pp. 1078-1083.

[37] H. K. Kim, Y. J. Kim, J. T. Kim, et al., "Alterations in the Proangiogenic Functions of Adipose Tissue-Derived Stromal Cells Isolated from Diabetic Rats," Stem Cells and Development, Vol. 17, No. 4, 2008, pp. 669-680. doi:10.1089/scd.2007.0141 
[38] Y. Lin, X. Chen, Z. Yan, et al., "Multilineage Differentiation of Adipose-Derived Stromal Cells from GFP Transgenic Mice," Molecular and Cellular Biochemistry, Vol. 285, No. 1-2, 2006, pp. 69-78. doi:10.1007/s11010-005-9056-8

[39] N. Krankel, V. Adams, A. Linke, et al., "Hyperglycemia Reduces Survival and Impairs Function of Circulating Blood-Derived Progenitor Cells," Arteriosclerosis, Thrombosis, and Vascular Biology, Vol. 25, No. 4, 2005, pp. 698-703. doi:10.1161/01.ATV.0000156401.04325.8f

[40] D. M. Breen, K. K. Chan, J. K. Dhaliwall, et al., "Insulin Increases Reendothelialization and Inhibits Cell Migration and Neointimal Growth after Arterial Injury," Arte- riosclerosis, Thrombosis, and Vascular Biology, Vol. 29, No. 7, 2009, pp. 1060-1066. doi:10.1161/ATVBAHA.109.185447

[41] W. Zhang, X. Wang, H. Jin, et al., "Effects of High Glucose Plus High Insulin on Proliferation and Apoptosis of Mouse Endothelial Progenitor Cells," Inflammation Research: Official Journal of the European Histamine Research Society, Vol. 57, 2008, pp. 571-576.

[42] M. E. Varney, W. E. Hardman and V. E. Sollars, “Omega 3 Fatty Acids Reduce Myeloid Progenitor Cell Frequency in the Bone Marrow of Mice and Promote Progenitor Cell Differentiation," Lipids in Health and Disease, Vol. 8, 2009, p. 9. doi:10.1186/1476-511X-8-9

\section{Additional}

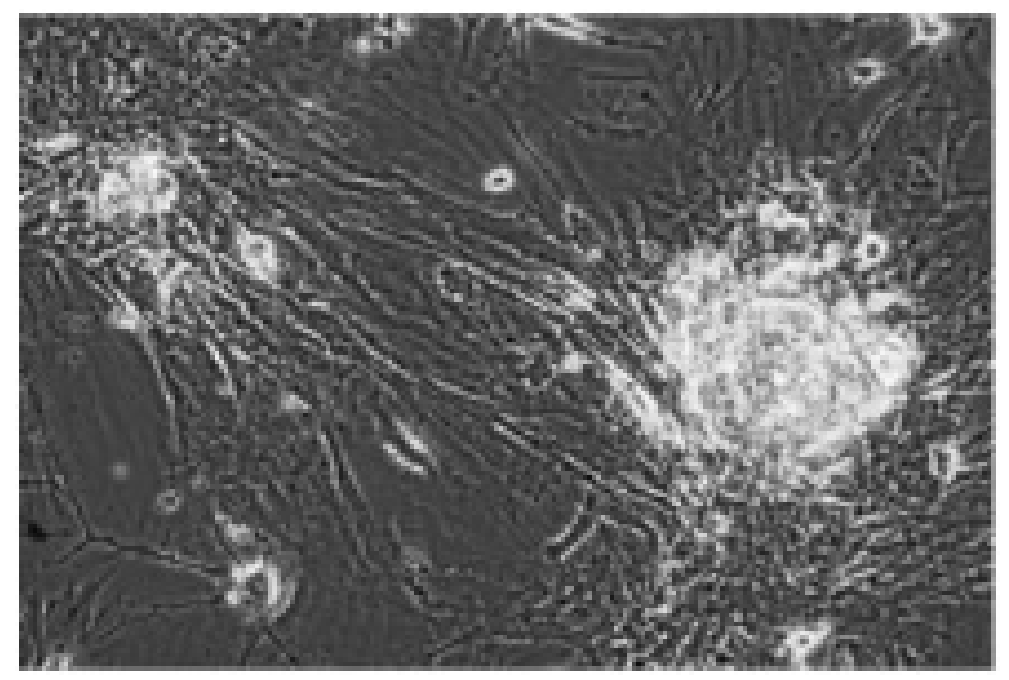

Figure S1. Arrangement of ASC into colony forming units 12 days post-plating onto collagen coated plates. 